Original Research

\title{
Early Nutrition during Critical Illness in Pediatric Patients Post- Hematopoietic Cell Transplantation
}

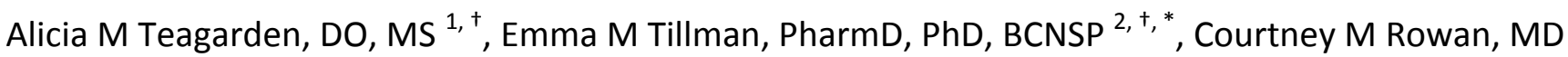
$1,+$

1. Department of Pediatrics, Section of Critical Care, Indiana University School of Medicine, Indianapolis, IN, USA; Emails: agranos@iupui.edu; coujohns@iu.edu

2. Department of Pharmacy, Riley Hospital for Children at Indiana University Health, Indianapolis, IN, USA; Email: etillman@iuhealth.org

† These authors contributed equally to this work

* Corresponding author: Emma M. Tillman; Email: etillman@iuhealth.org

Academic Editor: Mohammed Moghadasian

Special Issue: Diets and Transplantation

OBM Transplantation

2018, volume 2 , issue 4

doi:10.21926/obm.transplant.1804022
Received: April 24, 2018

Accepted: October 23, 2018

Published: October 29, 2018

\section{Abstract}

Background: Hematopoietic cell transplant (HCT) patients are a high-risk population for poor nutrition and decreased survival. There is little data on how nutrition in pediatric HCT patients affects need for critical care interventions and outcomes.

Methods: We hypothesized that patients who did not meet goal nutrition by 72 hours postadmission to the pediatric intensive care unit (PICU) had increased in-hospital mortality and increased need for critical care interventions. We performed a retrospective cohort study of pediatric HCT patients that were admitted to the PICU during their transplant admission.

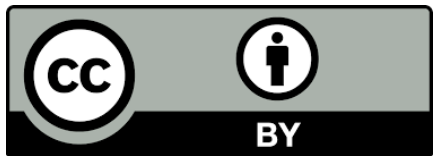

(C) 2018 by the author. This is an open access article distributed under the conditions of the Creative Commons by Attribution License, which permits unrestricted use, distribution, and reproduction in any medium or format, provided the original work is correctly cited. 
Patients were included if they were $<21$ years and post-allogeneic HCT. Goal nutrition was defined as the goal $\mathrm{kcal} / \mathrm{day}$ or $\mathrm{kcal} / \mathrm{kg} /$ day set by the dietician using the Schofield equation with appropriate age, gender, stress and activity factors. We compared patients who did not meet goal nutrition within 72 hours of PICU admission to those who met goal nutrition within 72 hours.

Results: Twenty-eight patients were included in the study and 54\% ( $n=15)$ did not meet goal nutrition by 72 hours from PICU admission. Those that did not meet goal nutrition by 72 hours had higher rates of in-hospital mortality (67\% vs $23 \%$; $\mathrm{p}=0.02$ ), increased 12 -month post-HCT mortality $(80 \%$ vs $31 \% ; p<0.01)$ and increased rates of veno-occlusive disease $(67 \%$ vs $15 \% ; p$ $<0.01)$.

Conclusions: We conclude that there is an association with poor nutrition in critically ill pediatric HCT patients and decreased survival. Further research is needed to evaluate the effect of improved nutrition at admission to the PICU and outcomes.

\section{Keywords}

Critical care; critical Illness; hematopoietic stem cell transplantation; intensive care units, pediatric; nutritional status

\section{Introduction}

Malnutrition occurs in $20-47 \%$ of critically ill children and is associated with poor outcomes [1-3]. These outcomes include longer pediatric intensive care unit (PICU) stays, prolonged mechanical ventilation, and increased mortality rates. It is known that critically ill pediatric patients have a high risk of malnutrition while in the PICU due in part to illness, inadequate delivery of nutrition, and unknown or poor nutritional status prior to admission [4]. Hematopoietic cell transplant (HCT) patients are a high-risk population for poor nutrition, which is known to be a negative predictive factor for outcomes [5-7]. These patients are also at high risk to have poor nutritional status prior to transplant. In fact, a recent study demonstrated that pediatric HCT patients with hypoalbuminemia in the pre-transplant period have been shown to have an increased need for critical care interventions and increased 6-month mortality [8].

Nutritional needs are increased in HCT patients both pre- and post-transplant because of a stressinduced catabolic state from infection, graft-versus-host disease (GVHD), or gut toxicity secondary to chemotherapy and irradiation [9]. Close attention to nutritional needs is important for preventing malnutrition from either gut injury leading to poor nutrient absorption or from increased nutritional requirements.

There is a wealth of data on the importance of early and aggressive nutrition support in both adult and pediatric HCT patients. However, there are little data looking at nutritional support in critically ill pediatric HCT patients upon admission to the PICU. It is also unknown how nutritional intervention affects critical care outcomes and mortality. We aimed to evaluate how early nutrition during critical 
illness in pediatric patients post-HCT is associated with outcomes. We hypothesized that patients who did not meet goal nutrition by 72 hours after admission to the PICU had increased need for critical care interventions and increased in-hospital mortality.

\section{Materials and Methods}

Institutional Review Board approval was obtained prior to start of the study. All procedures were followed in accordance with the ethical standards of the responsible institutional committee on human experimentation.

A retrospective cohort study was conducted of pediatric allogeneic HCT patients admitted to our PICU during their transplant admission between January 1, 2009 and December 31, 2014.

The primary objective was to investigate whether ability to meet goal nutrition within 72 hours of PICU admission was associated with improved critical care outcomes. Goal nutrition was defined as the goal kilocalorie (kcal)/day or goal $\mathrm{kcal} / \mathrm{kg} /$ day set by the dietician in the most recent nutrition note in the electronic medical record. Indirect calorimetry data were not performed on these patients and as this was a retrospective cohort study, we were unable to obtain these data. Instead, the Schofield Equation [10] was performed to calculate the goal nutrition for each specific patient using appropriate age, gender, activity and stress factors. The primary outcome measure was survival. Secondary outcomes included: need for critical care interventions, diagnosis of veno-occlusive disease (VOD) and diagnosis of graft-versus-host disease (GVHD). Critical care interventions were defined as the need for mechanical ventilation and/or renal replacement therapy. A sub-analysis was performed on patients evaluating no nutrition at 24 hours and outcomes.

\subsection{Patient Population}

Patients were included if they were $\leq 21$ years of age, post-allogeneic HCT, and if they were admitted to the PICU during their transplant admission. Data from subsequent PICU admissions during the transplant admission were excluded. Body mass index (BMI) and weight-for-length were obtained at transplant admission. We compared patients who did not meet goal nutrition by 72 hours of PICU admission to those who did meet goal nutrition within 72 hours.

\subsection{Data Collection}

Data were obtained from the electronic medical record and entered into Research Electronic Data Capture database [11]. The following demographic and transplant data were collected: age, gender, height and weight at transplant admission, indication for transplant, donor source (bone marrow, cord blood, or peripheral blood stem cells), and donor match. Nutrition data were collected at PICU day 0 (day of PICU admission) and for the subsequent 72 hours of PICU admission. We collected the total daily $\mathrm{kcal} / \mathrm{kg} / \mathrm{day}$ or $\mathrm{kcal} / \mathrm{day}$ and compared to the goal nutrition set by the dietician. Goal nutrition was met on the first day the patient received $100 \%$ of their patient specific calculated $\mathrm{kcal} / \mathrm{kg} /$ day or $\mathrm{kcal} /$ day goal as prescribed by the dietician. The outcome variables collected were 
diagnosis of VOD, diagnosis of GVHD, need for mechanical ventilation, need for any form of renal replacement therapy, in-hospital survival, and survival at 12 months post-HCT.

Based on the Centers for Disease Control and Prevention (CDC) nutrition surveillance guidelines, patients were defined as underweight at transplant admission if they were less than the $5^{\text {th }}$ percentile on their respective gender-specific growth charts [12]. Using the CDC recommendations, World Health Organization weight-for-length growth charts were used for infants and children ages 0 to 2 years of age, and CDC body mass index-for-age growth charts for children age 2 years and older [13].

\subsection{Statistical Analysis}

All patients were categorized into two groups: those that did not meet goal nutrition by 72 hours of PICU admission and those that met goal within 72 hours. Bivariate analyses for comparing these two groups were conducted for various demographic and clinical variables. Continuous variables are expressed as medians with interquartile range (IQR) and were compared using Wilcoxon rank sum test. Categorical variables are expressed as absolute counts with percentages, and were compared using Fisher exact test or chi-squared analysis as appropriate. The differences between groups were considered to be statistically significant with a $p$ value $<0.05$.

\section{Results}

A total of 28 patients met our inclusion criteria for the study. Demographics from the entire cohort, and then stratified based on whether or not they met goal nutrition at 72 hours of PICU admission, are displayed in Table 1. Gender, age, transplant demographic, and reason for PICU admission data did not differ significantly between groups. There was also no difference in weight categories at transplant admission and meeting goal nutrition at 72 hours $(p=0.69)$.

Table 2 displays the route of nutrition received at 72 hours. The groups were broken down into nutrition provided solely by the parenteral route, solely by an enteral route (including the use of a feeding tube), or a combination of parenteral and enteral nutrition. There was no significant difference between type of nutrition received and meeting goal nutrition at 72 hours.

Using the goal set by the dietician, 54\% $(n=15)$ did not meet goal nutrition by 72 hours from PICU admission. Those who did not meet goal nutrition by 72 hours from PICU admission had higher rates of in-hospital mortality (67\% vs $23 \% ; p=0.02$ ) and higher rates of 12 -month post-HCT mortality $(80 \%$ vs $31 \% ; p<0.01$ ). Those that did not meet goal nutrition by 72 hours also had higher rates of VOD ( $67 \%$ vs $15 \% ; p<0.01$ ). There was no statistical difference in the need for renal replacement therapy, need for mechanical ventilation, or diagnosis of GVHD (Table 3). There was also no difference in the median length of PICU stay (0.09), length of hospital stay (0.34), length of invasive mechanical ventilation $(p=0.29)$, and the length of vasoactive agent use $(p=0.19)$.

In our sub-analysis, we found that $21 \%(n=6)$ of patients did not receive any form of nutrition (including either parenteral nutrition or enteral nutrition) in the first 24 hours of PICU admission. Patients that did not receive any form of nutrition in the first 24 hours had greater need for renal replacement therapy compared to those that received any amount of nutrition in 24 hours (67\% vs 
$18 \% ; p=0.04$ ). In addition, patients that did not receive any nutrition in 24 hours had higher rates of VOD compared to those that received some form of nutrition ( $83 \%$ vs $32 \% ; p=0.05$ ).

Table 1 Baseline characteristics for all patients stratified by nutrition intake at 72 hours.

\begin{tabular}{|c|c|c|c|c|}
\hline & $\begin{array}{l}\text { Entire Cohort } \\
(n=28)\end{array}$ & $\begin{array}{l}\text { Met goal nutrition } \\
\leq 72 \text { hours } \\
(n=13)\end{array}$ & $\begin{array}{l}\text { Did not meet goal } \\
\text { nutrition } \leq 72 \text { hours } \\
(n=15)\end{array}$ & $p$ value \\
\hline Gender (female), n (\%) & $11(39)$ & $5(38)$ & $6(40)$ & 0.93 \\
\hline Age (years), (IQR) & $4(0.25,12)$ & $4(0,12)$ & $4(0,12)$ & 0.71 \\
\hline Diagnosis, n (\%) & & & & 0.21 \\
\hline AML & $5(18)$ & $3(22)$ & $2(13)$ & \\
\hline ALL & $7(25)$ & $4(31)$ & $3(20)$ & \\
\hline $\mathrm{HLH}$ & $4(14)$ & $0(0)$ & $4(27)$ & \\
\hline Immunodeficiencies & $5(18)$ & $4(31)$ & $1(7)$ & \\
\hline Lymphomas & $2(7)$ & $1(8)$ & $1(7)$ & \\
\hline Bone marrow failure & $2(7)$ & $0(0)$ & $2(13)$ & \\
\hline Others & $3(11)$ & $1(8)$ & $2(13)$ & \\
\hline Donor source, $\mathrm{n}(\%)$ & & & & 0.39 \\
\hline Bone marrow & $9(32)$ & $5(38)$ & $4(27)$ & \\
\hline Cord blood & $18(64)$ & $7(54)$ & $11(73)$ & \\
\hline Peripheral blood & $1(4)$ & $1(8)$ & $0(0)$ & \\
\hline Matched, n (\%) & $17(61)$ & $7(54)$ & $10(83)$ & 0.21 \\
\hline $\begin{array}{l}\text { Weight categories at } \\
\text { transplant admission, } \mathrm{n}(\%)\end{array}$ & & & & 0.69 \\
\hline Underweight, n (\%) & $4(14)$ & $2(15)$ & $2(13)$ & \\
\hline Normal weight, n (\%) & $13(46)$ & $7(54)$ & $6(40)$ & \\
\hline Overweight/Obese, n (\%) & $11(40)$ & $4(31)$ & $7(47)$ & \\
\hline PICU admit diagnosis, n (\%) & & & & 0.91 \\
\hline Respiratory failure, $\mathrm{n}(\%)$ & $19(68)$ & $8(62)$ & $11(74)$ & \\
\hline Sepsis/Septic shock, n (\%) & $4(14)$ & $2(15)$ & $2(13)$ & \\
\hline Seizures/AMS, n (\%) & $4(14)$ & $2(15)$ & $2(13)$ & \\
\hline Fluid overload, n (\%) & $1(4)$ & $1(8)$ & $0(0)$ & \\
\hline
\end{tabular}

$A L L=$ acute lymphoblastic leukemia, $A M L=$ acute myeloid leukemia, AMS = altered mental status, $\mathrm{HLH}=$ hemophagocytic lymphohistiocytosis.

Categorical variables are expressed as frequencies with (\%) of the patient numbers in the respective column. Continuous variables are presented as median with interquartile range (IQR). 
Table 2 Route of nutrition received at 72 hours for all patients.

\begin{tabular}{|l|l|l|l|}
\hline & $\begin{array}{l}\text { Entire Cohort } \\
(\mathbf{n}=\mathbf{2 8})\end{array}$ & $\begin{array}{l}\text { Met goal nutrition } \\
\mathbf{5 7 2} \text { hours } \\
(\mathbf{n}=\mathbf{1 3 )}\end{array}$ & $\begin{array}{l}\text { Did not meet goal } \\
\text { nutrition } \leq 72 \text { hours } \\
\text { (n= 15) }\end{array}$ \\
\hline Parenteral nutrition only & $23(82)$ & $11(86)$ & $12(80)$ \\
\hline Enteral nutrition only & $3(11)$ & $1(7)$ & $2(13)$ \\
\hline $\begin{array}{l}\text { Combination of parenteral } \\
\text { and enteral nutrition }\end{array}$ & $2(7)$ & $1(7)$ & $1(7)$ \\
\hline
\end{tabular}

* No significant difference in route of nutrition with regards to achieving goal nutrition at 72 hours.

Categorical variables are expressed as frequencies with (\%) of the patient numbers in the respective column.

Table 3 Critical care interventions assessed by nutritional intake at 72 hours.

\begin{tabular}{|c|c|c|c|c|}
\hline Critical care interventions & $\begin{array}{l}\text { Entire cohort } \\
(n=28)\end{array}$ & $\begin{array}{l}\text { Met goal nutrition } \\
\leq 72 \text { hours } \\
\text { ( } n=13)\end{array}$ & $\begin{array}{l}\text { Did not meet } \\
\text { goal nutrition } \\
\leq 72 \text { hours } \\
\text { ( } \mathrm{n}=15)\end{array}$ & $p$ value \\
\hline Noninvasive ventilation, $\mathrm{n}(\%)$ & $11(39.3)$ & $7(53.8)$ & $4(26.7)$ & 0.25 \\
\hline $\begin{array}{l}\text { Invasive mechanical } \\
\text { ventilation, } \mathrm{n}(\%)\end{array}$ & $23(82.1)$ & $11(84.6)$ & $12(80.0)$ & $>0.99$ \\
\hline $\begin{array}{l}\text { Renal replacement therapy, } \mathrm{n} \\
(\%)\end{array}$ & $8(28.6)$ & $2(15.4)$ & $6(40.0)$ & 0.22 \\
\hline Vasoactive agent use, n (\%) & $16(57.1)$ & $8(61.5)$ & $8(53.3)$ & 0.66 \\
\hline
\end{tabular}

Values displayed are frequency (\%) and were compared using chi squared or Fisher's Exact tests where appropriate.

\section{Discussion}

Our data suggest that poor nutrition in critically ill pediatric HCT patients is associated with both inhospital mortality and 12-month mortality. To our knowledge, there are no published studies evaluating acceptable time to goal nutrition in critically ill pediatric HCT patients. However, there are several studies that have evaluated time to initiation of feeds in critically ill children. In the general PICU population, studies have shown that failure to achieve goal nutrition or underfeeding has a negative effect on patient outcomes. In a prospective, multicenter, cohort study, Mehta et al. evaluated the relationship between protein intake and 60-day mortality in 1254 mechanically ventilated (MV) PICU patients from 59 centers. They found that adequate enteral protein intake was significantly associated with decreased 60-day mortality [14]. Mikhaliov et al, evaluated early enteral nutrition defined as meeting $25 \%$ of goal enteral nutrition within 48 hours of PICU admission in 5105 
children. They concluded that early enteral nutrition is associated with decreased mortality in patients with PICU length of stay of $\geq 96$ hours [15].

The critically ill HCT population is an important population to study, as it has been shown that need for critical care interventions in this population is associated with high mortality rates [16-18]. Factors that have been shown to be significant predictors of poor outcome in the HCT population include need for dialysis during the ICU stay or respiratory failure [19]. HCT recipients that require invasive mechanical ventilation have been found to have PICU survival rates ranging between $40 \%$ and $60 \%$ $[17,18,20]$. Little investigation has been done on the effect of poor nutrition on the need for critical care interventions. With this high mortality rate, there is tremendous opportunity to improve outcomes. Thus, a focus on nutritional intervention may be in order.

In addition to demonstrating that poor nutrition may contribute to decreased PICU survival, we also found an association with poor nutrition and complications including diagnosis of VOD and need for renal replacement therapy. It has been shown that renal replacement therapy is associated with increased mortality in the pediatric HCT population [21]. Our findings have lead us to question if we are sacrificing nutrition in order to achieve optimal fluid balance in the critically ill pediatric HCT population. Fluid balance in the PICU remains a challenge to delicately balance successful fluid resuscitation while avoiding fluid overload, which can contribute to kidney, respiratory, and cardiac complications. In order to achieve an even balance or negative fluid status, fluids including nutrition may be restricted. Sutherland et al, studied the association between fluid overload and mortality in pediatric patients receiving continuous renal replacement therapy. They found that critically ill children that develop greater fluid overload before initiation of renal replacement therapy experienced higher mortality rates than those with less fluid overload [22]. Thus, further research needs to be conducted evaluating improving nutrition while balancing fluid administration in the critically ill pediatric HCT population.

For our study, we chose 72 hours post-admission to the PICU as a timeframe for reaching goal nutrition as this is a reasonable length of time in our critically ill children. This allows for patients who require vasopressor support, mechanical ventilation, and/or renal replacement therapy to slowly titrate up on nutrition. Even if patients are receiving trophic feeds initially, advancing to goal within 72 hours is a reasonable timeframe. Surprisingly, we found that $21 \%(n=6)$ of our patients received no nutrition within the first 24 hours. This is likely due to the fact that those 24 hours are spent in the acute resuscitation of our critically ill HCT patients, whether it be managing septic shock or providing more respiratory support. However, it has been shown that there are multiple benefits of nutrition support in the critically ill population, including improvement in wound healing, reducing catabolic response to injury and sepsis, and enhancing gastrointestinal function [23]. Thus, the lack of any nutrition in 24 hours should serve as a red flag for providers and thought should be given to starting early nutrition in our critically ill patients.

We also found that only $18 \%$ of our total population of patients received any form of enteral nutrition at 72 hours of PICU admission. This is surprising, as studies have shown the importance of enteral nutrition during critical illness as a benefit for gastrointestinal mucosal integrity and motility [24]. In fact, recent guidelines published by the Society of Critical Care Medicine and American Society for Parenteral and Enteral Nutrition recommend enteral nutrition as the preferred mode of nutrition 
delivery to the critically ill child. These guidelines also mention that enteral nutrition can be safely delivered to critically ill children with medical and surgical diagnoses as well as those receiving vasoactive medications [25].

Our study is limited by the relatively small sample size along with the retrospective, single center design. Our study also lacks an objective measure of nutritional status (i.e. indirect calorimetry or nitrogen balance). Instead, we used the dietician recommendations for goal daily calories as calculated by the Schofield Equation which was used to calculate the goal nutrition for each specific patient using appropriate age, gender, activity and stress factors. In addition, we did not look at nutritional status leading up to the PICU admission because this is not always available for our patients as this was a retrospective study. We also did not analyze PICU admission weights because of the likely fluid balance and capillary leak in these critically ill patients. This fluid balance and capillary leak would likely be confounders. Despite these limitations, we believe our results spark interesting questions that could impact success of HCT. Nutritional status should be carefully evaluated during the pre-HCT evaluation and when possible optimal nutritional status should be achieved prior to initiating conditioning for HCT.

\section{Conclusion}

Our data in this small retrospective pilot study have shown that poor nutrition in critically ill pediatric HCT patients may increase morbidity and mortality. Further research is needed to evaluate improved nutrition at admission to the PICU and outcomes of these critically ill pediatric HCT patients. Ultimately, data from these nutrition studies may be used to develop a standardized nutrition protocol for critically ill pediatric HCT patients.

\section{Acknowledgements}

None

\section{Author Contributions}

Drs. Teagarden, Tillman and Rowan all contributed to the (i) concept and design, or acquisition of data, or analysis and interpretation of data; (ii) drafting the article or revising it critically for important intellectual content; and (iii) final approval of the version to be published.

\section{Funding}

No financial or nonfinancial benefits have been received or will be received from any party related directly or indirectly to the subject of this article.

\section{Competing Interests}

None declared for all authors. 


\section{References}

1. de Souza Menezes F, Leite HP, Koch Nogueira PC. Malnutrition as an independent predictor of clinical outcome in critically ill children. Nutrition. 2012; 28: 267-270.

2. Martinez EE, Mehta NM. The science and art of pediatric critical care nutrition. Curr Opin Crit Care. 2016; 22: 316-324.

3. Mehta NM, Bechard LJ, Cahill N, Wang M, Day A, Duggan CP, et al. Nutritional practices and their relationship to clinical outcomes in critically ill children--an international multicenter cohort study*. Crit Care Med. 2012; 40: 2204-2211.

4. Zamberlan P, Delgado AF, Leone C, Feferbaum R, Okay TS. Nutrition therapy in a pediatric intensive care unit: indications, monitoring, and complications. JPEN J Parenter Enteral Nutr. 2011; 35: 523-529.

5. Martin-Salces M, de Paz R, Canales MA, Mesejo A, Hernandez-Navarro F. Nutritional recommendations in hematopoietic stem cell transplantation. Nutrition. 2008; 24: 769-775.

6. Raynard B, Nitenberg G, Gory-Delabaere G, Bourhis JH, Bachmann P, Bensadoun RJ, et al. Summary of the standards, options and recommendations for nutritional support in patients undergoing bone marrow transplantation (2002). Br J Cancer. 2003; 89: S101-106.

7. White $M$, Murphy AJ, Hastings $Y$, Shergold J, Young J, Montgomery $C$, et al. Nutritional status and energy expenditure in children pre-bone-marrow-transplant. Bone Marrow Transplant. 2005; 35: 775-779.

8. Teagarden AM, Skiles JL, Beardsley AL, Hobson MJ, Moser EAS, Renbarger JL, et al. Low serum albumin levels prior to pediatric allogeneic HCT are associated with increased need for critical care interventions and increased 6-month mortality. Pediatr Transplant. 2017; 21. doi: 10.1111/petr.13016.

9. Muscaritoli M, Grieco G, Capria S, Iori AP, Rossi Fanelli F. Nutritional and metabolic support in patients undergoing bone marrow transplantation. Am J Clin Nutr. 2002; 75: 183-190.

10. Schofield WN. Predicting basal metabolic rate, new standards and review of previous work. Hum Nutr Clin Nutr. 1985; 39 Suppl 1: 5-41.

11. Harris PA, Taylor R, Thielke R, Payne J, Gonzalez N, Conde JG. Research electronic data capture (REDCap)--a metadata-driven methodology and workflow process for providing translational research informatics support. J Biomed Inform. 2009; 42: 377-381.

12. Centers for Disease Control and Prevention. PedNSS Health Indicators [updated October 29, 2009. Available from: http://www.cdc.gov/pednss/what is/pednss health indicators.htm.

13. Centers for Disease Control and Prevention. WHO Growth Standards Are Recommended for Use in the U.S. for Infants and Children 0 to 2 Years of Age [updated September 9, 2010. Available from: http://www.cdc.gov/growthcharts/index.htm.

14. Mehta NM, Bechard $\amalg$, Zurakowski D, Duggan CP, Heyland DK. Adequate enteral protein intake is inversely associated with 60-d mortality in critically ill children: a multicenter, prospective, cohort study. Am J Clin Nutr. 2015; 102: 199-206. 
15. Mikhailov TA, Kuhn EM, Manzi J, Christensen M, Collins M, Brown AM, et al. Early enteral nutrition is associated with lower mortality in critically ill children. JPEN J Parenter Enteral Nutr. 2014; 38: 459-466.

16. Duncan CN, Lehmann LE, Cheifetz IM, Greathouse K, Haight AE, Hall MW, et al. Clinical outcomes of children receiving intensive cardiopulmonary support during hematopoietic stem cell transplant. Pediatr Crit Care Med. 2013; 14: 261-267.

17. Rowan CM, Gertz SJ, McArthur J, Fitzgerald JC, Nitu ME, Loomis A, et al. Invasive Mechanical Ventilation and Mortality in Pediatric Hematopoietic Stem Cell Transplantation: A Multicenter Study. Pediatr Crit Care Med. 2016; 17: 294-302.

18. van Gestel JP, Bollen CW, van der Tweel I, Boelens JJ, van Vught AJ. Intensive care unit mortality trends in children after hematopoietic stem cell transplantation: a meta-regression analysis. Crit Care Med. 2008; 36: 2898-2904.

19. Kache S, Weiss IK, Moore TB. Changing outcomes for children requiring intensive care following hematopoietic stem cell transplantation. Pediatric Transplant. 2006; 10: 299-303.

20. Chima RS, Daniels RC, Kim MO, Li D, Wheeler DS, Davies SM, et al. Improved outcomes for stem cell transplant recipients requiring pediatric intensive care. Pediatr Crit Care Med. 2012; 13: e336-342.

21. van Gestel JP, Bierings MB, Dauger $S$, Dalle JH, Pavlicek $P$, Sedlacek $P$, et al. Outcome of invasive mechanical ventilation after pediatric allogeneic hematopoietic SCT: results from a prospective, multicenter registry. Bone Marrow Transplant. 2014; 49: 1287-1292.

22. Sutherland SM, Zappitelli M, Alexander SR, Chua AN, Brophy PD, Bunchman TE, et al. Fluid overload and mortality in children receiving continuous renal replacement therapy: the prospective pediatric continuous renal replacement therapy registry. Am J Kidney dis. 2010; 55 : 316-325.

23. Wray CJ, Mammen JM, Hasselgren PO. Catabolic response to stress and potential benefits of nutrition support. Nutrition. 2002; 18: 971-977.

24. Lin MT, Saito H, Fukushima R, Inaba T, Fukatsu K, Inoue T, et al. Route of nutritional supply influences local, systemic, and remote organ responses to intraperitoneal bacterial challenge. Ann Surg. 1996; 223: 84-93.

25. Mehta NM, Skillman HE, Irving SY, Coss-Bu JA, Vermilyea S, Farrington EA, et al. Guidelines for the provision and assessment of nutrition support therapy in the pediatric critically III patient: society of critical care medicine and American society for parenteral and enteral nutrition. Pediatr Crit Care Med. 2017; 18: 675-715. 


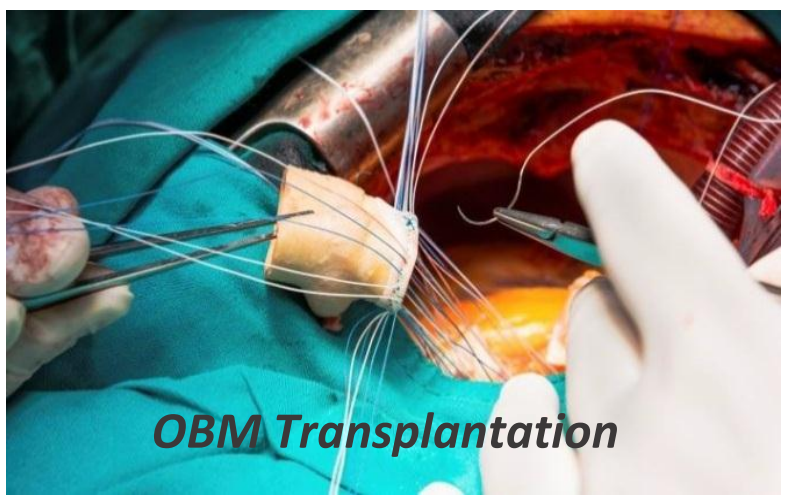

Enjoy $O B M$ Transplantation by:

1. Submitting a manuscript

2. Joining in volunteer reviewer bank

3. Joining Editorial Board

4. Guest editing a special issue

For more details, please visit:

http://www.lidsen.com/journals/transplantation 